\title{
Case Report: Lung Ultrasound for the Guidance of Adjunctive Therapies in Two Invasively Ventilated Patients with COVID-19
}

\author{
Charalampos Pierrakos, ${ }^{1,2 \star}$ Rachid Attou, ${ }^{1}$ Enrica lesu, ${ }^{3}$ Hugues Baelongandi, ${ }^{3}$ Patrick M. Honore, ${ }^{1}$ Lieuwe D. J. Bos, ${ }^{2,4,5}$ \\ Marcus J. Schultz, ${ }^{2,4,6,7}$ and David De Bels ${ }^{1}$ \\ ${ }^{1}$ Department of Intensive Care, Brugmann University Hospital, Université Libre de Bruxelles, Brussels, Belgium; ${ }^{2}$ Department of Intensive Care, \\ Amsterdam UMC, University of Amsterdam, Amsterdam, The Netherlands; ${ }^{3}$ Department of Anesthesiology, Brugmann University Hospital, \\ Université Libre de Bruxelles, Brussels, Belgium; ${ }^{4}$ Laboratory of Experimental Intensive Care and Anesthesiology (LEICA), Amsterdam UMC, \\ University of Amsterdam, Amsterdam, The Netherlands; ${ }^{5}$ Department of Respiratory Medicine, Amsterdam UMC, University of Amsterdam, \\ Amsterdam, The Netherlands; ${ }^{6}$ Mahidol-Oxford Tropical Medicine Research Unit (MORU), Mahidol University, Bangkok, Thailand; ${ }^{7}$ Nuffield \\ Department of Medicine, University of Oxford, Oxford, United Kingdom
}

\begin{abstract}
Two patients with respiratory failure due to confirmed COVID-19 were examined using bedside lung ultrasound (LUS) shortly after intubation and start of invasive ventilation. In the first patient, LUS revealed extensive atelectatic areas. A recruitment maneuver was applied, resulting in some reaeration of areas that showed atelectasis, and some improvement in oxygenation was observed. Oxygenation improved further with the use of prone positioning. In the second patient, LUS showed diffuse abnormalities without atelectatic areas, and ventilation proceeded without a recruitment maneuver but with prone positioning. These two cases illustrate how LUS could be useful in identifying different lung morphologies early after the start of invasive ventilation and help decide on adjunctive therapies. This has possible implications for ventilator management in resource-limited settings, with limited availability of chest computed tomography and blood gas analyzers. Tailoring invasive ventilation based on LUS findings early after the start of invasive ventilation is feasible, but this should be further evaluated in future studies.
\end{abstract}

\section{INTRODUCTION}

The world is increasingly struggling with the COVID-19 pandemic, causing tens of thousands of hospitalizations worldwide each week. In many low- and middle-income countries, these numbers are rising rapidly. ${ }^{1}$ An estimated $5 \%$ of hospitalized patients will need admission to an intensive care unit (ICU), mainly for receiving invasive ventilation, ${ }^{2}$ and a personalized ventilator approach may be needed. ${ }^{3}$ Chest computed tomography (CT) has been proposed to guide the assessment of reaeration, given that surrogate markers like global oxygenation and respiratory system compliance might be misleading. ${ }^{4}$ Lung ultrasound (LUS), a noninvasive imaging technique that can be used at the bedside, is an attractive alternative to chest $\mathrm{CT},{ }^{5}$ especially in settings where resources are restricted. Here, we present two cases of COVID19 in which LUS contributed to the assessment of loss of aeration of lung tissue.

The global LUS score for semi-quantification of lung aeration. One frequently used tool to quantify extend of pulmonary pathologies is the so-called global LUS score, in which LUS patterns across 12 lung regions are caught in a numerical score. ${ }^{5} \mathrm{An}$ "A-pattern" (i.e., repeating horizontal [A-] lines parallel to the pleural line, suggesting normal aeration) is scored "0"; a "B-pattern" (i.e., three or more vertical [B-] lines starting from the pleural line and reaching the bottom of the screen, suggesting partial loss of aeration) is scored " 1 " if lines are well spaced, and "2" if lines are confluent; and a "C-pattern" (i.e., consolidation, suggesting near-complete to complete loss of aeration) is scored " 3 ." The individual scores per region are summed into a global LUS aeration score, which thus ranges

\footnotetext{
*Address correspondence to Charalampos Pierrakos, Department of Intensive Care, Brugmann University Hospital, A. van Gehuchtenplein 4, Brussel 1020, Belgium. E-mail: charalampos.pierrakos@chubrugmann.be
}

from "0" (normal aeration in all regions) to " 36 " (severe abnormal aeration in all regions).

\section{CASE REPORTS}

Patient A, a 66-year-old woman with a body mass index of $36 \mathrm{~kg} / \mathrm{m}^{2}$, was intubated 10 days after hospitalization for reverse transcriptase-PCR (RT-PCR)-confirmed COVID-19, and 3 days after admission to the ICU. The chest CT obtained on the day of hospitalization showed typical groundglass opacities with interlobular and intralobular septal thickening ("crazy paving") involving $30 \%$ of the lungs (Figure 1; panel A). Two days before the start of invasive ventilation, the global LUS score was 15 , with none of the examined regions scoring "3."

Invasive ventilation started with a tidal volume of $7 \mathrm{~mL} / \mathrm{kg}$ predicted body weight, $10 \mathrm{~cm} \mathrm{H}_{2} \mathrm{O}$ positive end-expiratory pressure (PEEP), and $100 \% \mathrm{FiO}_{2}$. Oxygenation was poor, with a $\mathrm{PaO}_{2}$ to $\mathrm{FiO}_{2}$ ratio of $61 \mathrm{mmHg}$, and the pulmonary system appeared stiff, with a compliance of $24 \mathrm{~mL} / \mathrm{cm} \mathrm{H}_{2} \mathrm{O}$ at a driving pressure of $14 \mathrm{~cm} \mathrm{H}_{2} \mathrm{O}$. At that moment, the global LUS score was 17 , with more regions scoring " 3 " at the dorsal site (Figure 2; panel 1). A significant recruitment maneuver, consisting of continuous airway pressure at $60 \mathrm{~cm} \mathrm{H} \mathrm{H}_{2} \mathrm{O}$ for 40 seconds, resulted in some improvement in the global LUS score from 17 to 14 , mainly through improvements in aeration of dorsal areas (Figure 2; panel 2 and Figure 3). An increase in oxygen saturation from $88 \%$ to $100 \%$ was observed, although need for high $\mathrm{FiO}_{2}$ remained. Prone positioning was applied, resulting in a sturdy improvement in the $\mathrm{PaO}_{2}$ to $\mathrm{FiO}_{2}$ ratio from 120 to $270 \mathrm{mmHg}$ and an increase in the pulmonary system compliance from 24 to $30 \mathrm{~mL} / \mathrm{cm} \mathrm{H}_{2} \mathrm{O}$ at a driving pressure of $11 \mathrm{~cm} \mathrm{H}_{2} \mathrm{O}$.

Patient $\mathrm{B}$, a 60 -year-old man with a body mass index of $37 \mathrm{~kg} / \mathrm{m}^{2}$, was intubated 12 days after hospitalization for RTPCR-confirmed COVID-19, and 6 days after ICU admission. A chest CT performed on the day of hospitalization showed 

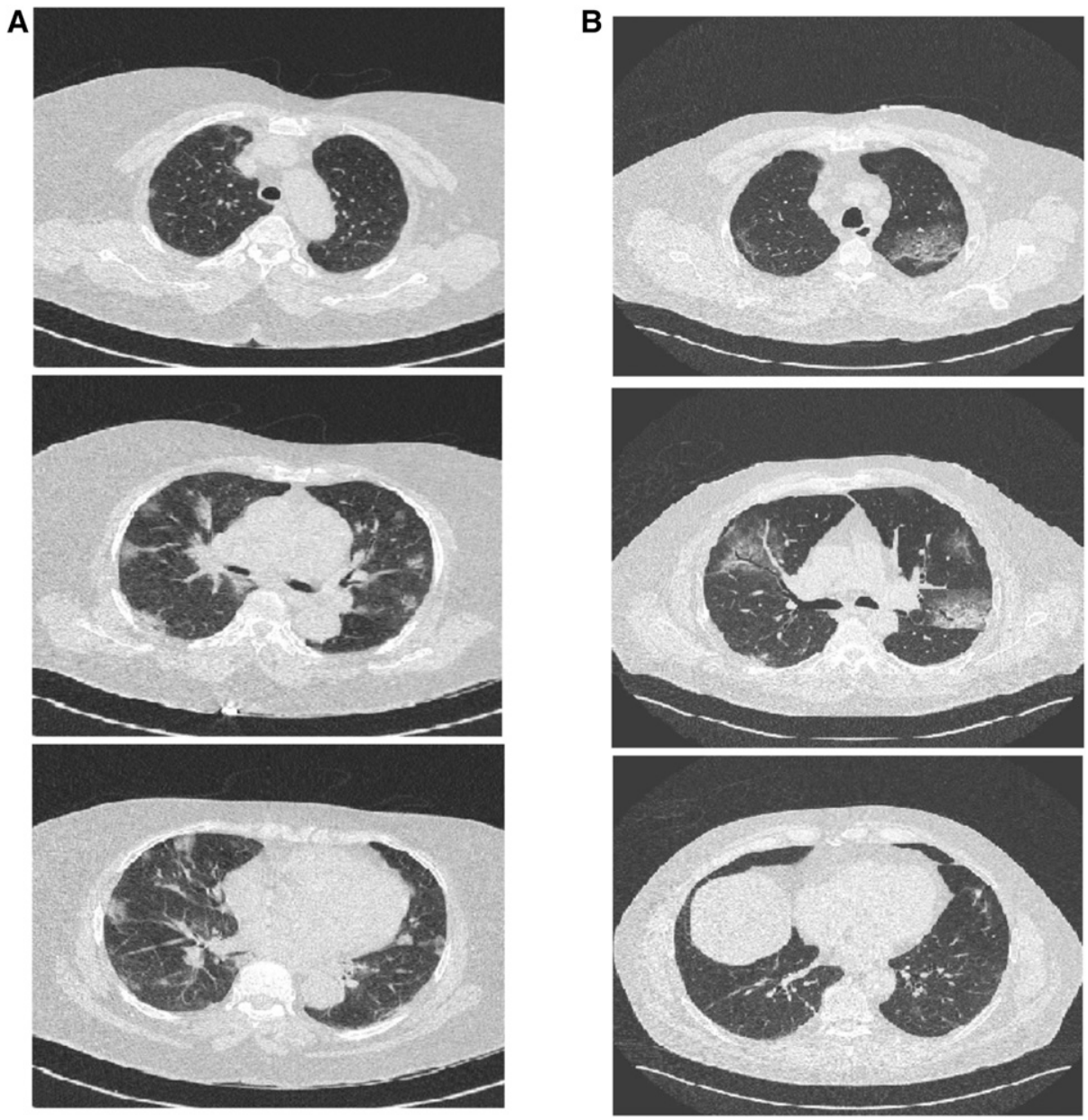

FIGURE 1. Chest computed tomography on the day of hospital admission for patients A (left) and B (right) at 10 and 12 days before intubation.

typical ground-glass and crazy paving involving $30 \%$ of the lungs (Figure 1, panel B). One day before intubation, the global LUS score was 11, with none of the examined areas scoring "3."

Invasive ventilation started with a tidal volume of $6 \mathrm{~mL} / \mathrm{kg}$ predicted body weight, $10 \mathrm{~cm} \mathrm{H}_{2} \mathrm{O}$ PEEP, and $100 \%$ oxygen. Oxygenation was poor with $\mathrm{PaO}_{2}$ to $\mathrm{FiO}_{2}$ ratio of $116 \mathrm{mmHg}$, and the respiratory system appeared stiff with a compliance of $40 \mathrm{~mL} / \mathrm{cm} \mathrm{H}_{2} \mathrm{O}$ at a driving pressure of $11 \mathrm{~cm} \mathrm{H}_{2} \mathrm{O}$. At the start of invasive ventilation, the global LUS score was 9 , with no single region scoring " 3 " (Figure 4). Ventilator settings were not changed, recruitment maneuvers were not performed, and the patient was placed in the prone position. After 16 hours of ventilation with $15 \mathrm{~cm} \mathrm{H}_{2} \mathrm{O}$ PEEP in the prone position, oxygenation had improved, with a rise in the $\mathrm{PaO}_{2}$ to $\mathrm{FiO}_{2}$ ratio from 116 to $150 \mathrm{mmHg}$. Pulmonary system compliance had not changed, $37 \mathrm{~mL} / \mathrm{cm} \mathrm{H}_{2} \mathrm{O}$ at a driving pressure was of $13 \mathrm{~cm} \mathrm{H} \mathrm{H}_{2} \mathrm{O}$.

\section{DISCUSSION}

These two case reports of COVID-19 illustrate the following: 1) LUS can be used to identify the lung morphological pattern of loss of aeration at the start of invasive ventilation, 2) differences in LUS patterns observed between these two patients included extent (i.e., a difference in the global LUS aeration score) and location (in patient A mainly located at the dorsal side) of loss of aeration, and 3) LUS could help in the decision to apply adjunctive therapies, especially the latter finding may have meaning for low-resource settings.

Several reports have shown the usefulness of LUS as a diagnostic tool in COVID-19. ${ }^{6-9}$ The findings in the cases described here point to the possibility to use LUS as a prediction tool for the effectiveness of adjunctive therapies. The patient with extensive atelectasis at the dorsal site responded poorly to a significant recruitment maneuver but responded well to prone positioning. The patient with less-extensive abnormalities also responded well to prone positioning. These findings are in accordance with those in a previous study that suggest that possibly patients with a focal morphology characterized by lobar, posterior atelectasis are more likely to be benefitted from prone position than from recruitment maneuvers. ${ }^{10}$

In these two cases, a recruitment maneuver and prone positioning were used as "rescue" therapies for refractory and life-threatening hypoxemia. Improvement in oxygenation, however, should never be considered as goal in itself, as improvement in oxygenation may not associated with a better outcome. ${ }^{11,12}$

Although in patient A oxygenation improved after the recruitment maneuver, only a minimal improvement in the global lung aeration was seen. Seen the fact that lung compliance 

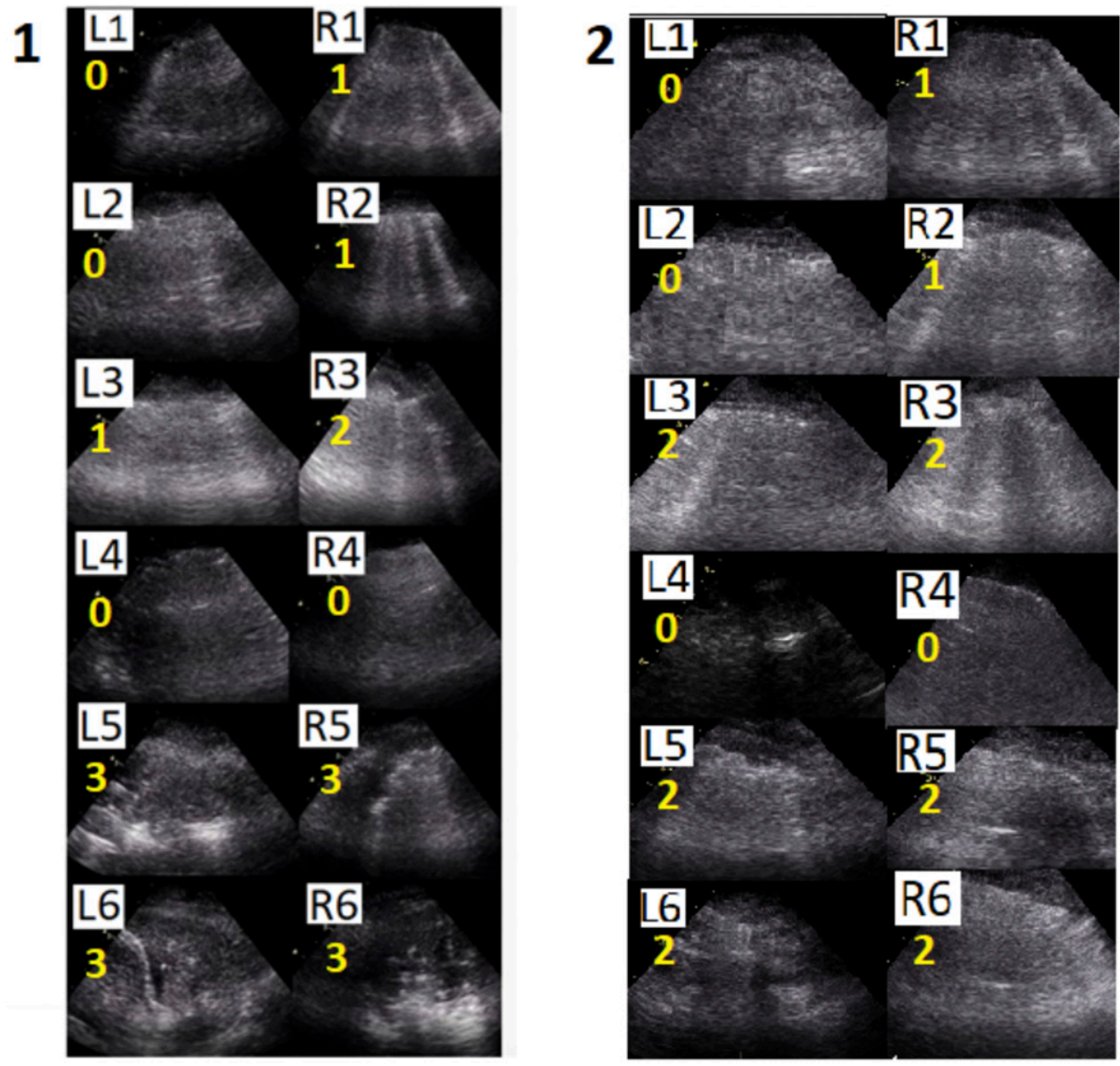

FIGURE 2. Lung ultrasound images for patient $A$, before (panel 1) and after the recruitment maneuver (panel 2). For each image, the corresponding score is presented $(0-3) . L=$ left hemithorax; $R=$ right hemithorax; $1,2=$ anterior thoracic areas; $3,4=$ lateral thoracic areas; $5,6=$ posterior thoracic areas.

remained low, hyperinflation may have occurred, which cannot be detected with LUS. ${ }^{13,14}$ Furthermore, regions with a "Cpattern" were located exclusively on the dorsal site that is not easily recruitable. COVID-19 patients with extensive consolidations in dorsal lung parts may thus respond better to prone positioning than to recruitment maneuvers. It should be noted that patient $B$ did not receive a recruitment maneuver, and we can only conclude that prone positioning leads to a satisfying improvement in oxygenation.
Lung ultrasound is attractive because ultrasound machines are widely available, mostly portably, and thereby the technique can be used even in resource-limited settings and eliminates the need for transport to the radiology department, for example, for CT scanning. ${ }^{15,16}$ Furthermore, LUS can be repeated many times as it is a bedside, radiation-free imaging technique. Of importance, repetition of lung imaging might be relevant in the context of COVID-19 as the morphology of the lung may change over time. Last but not least, this advantage
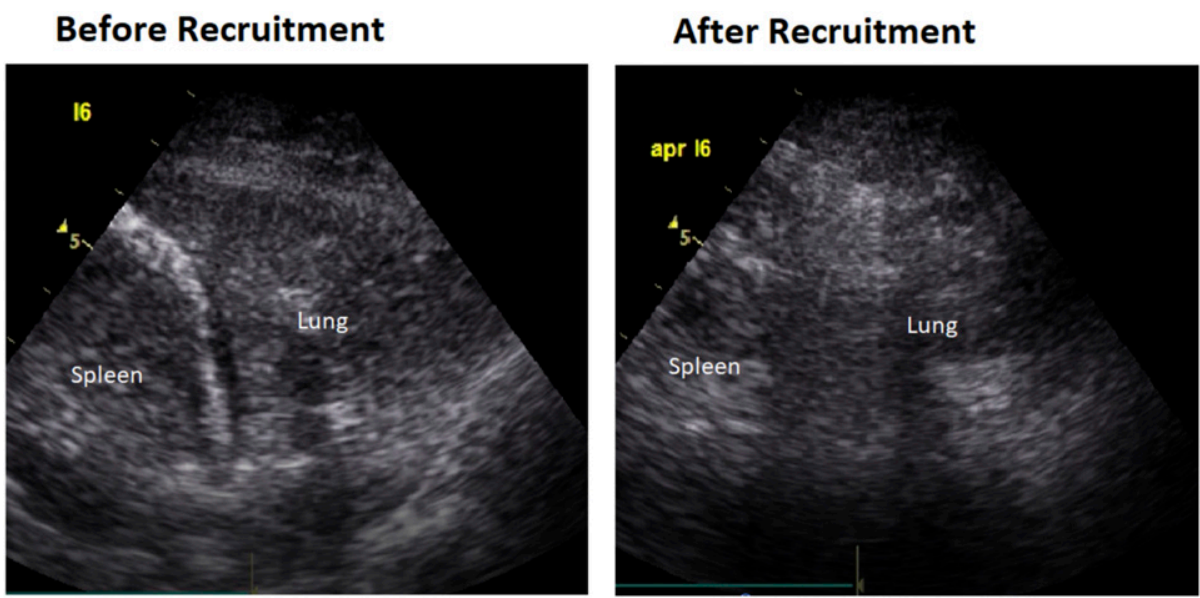

FIGURE 3. The effect of using recruitment maneuvers on the left posterior areas of the lung in patient $A$. 


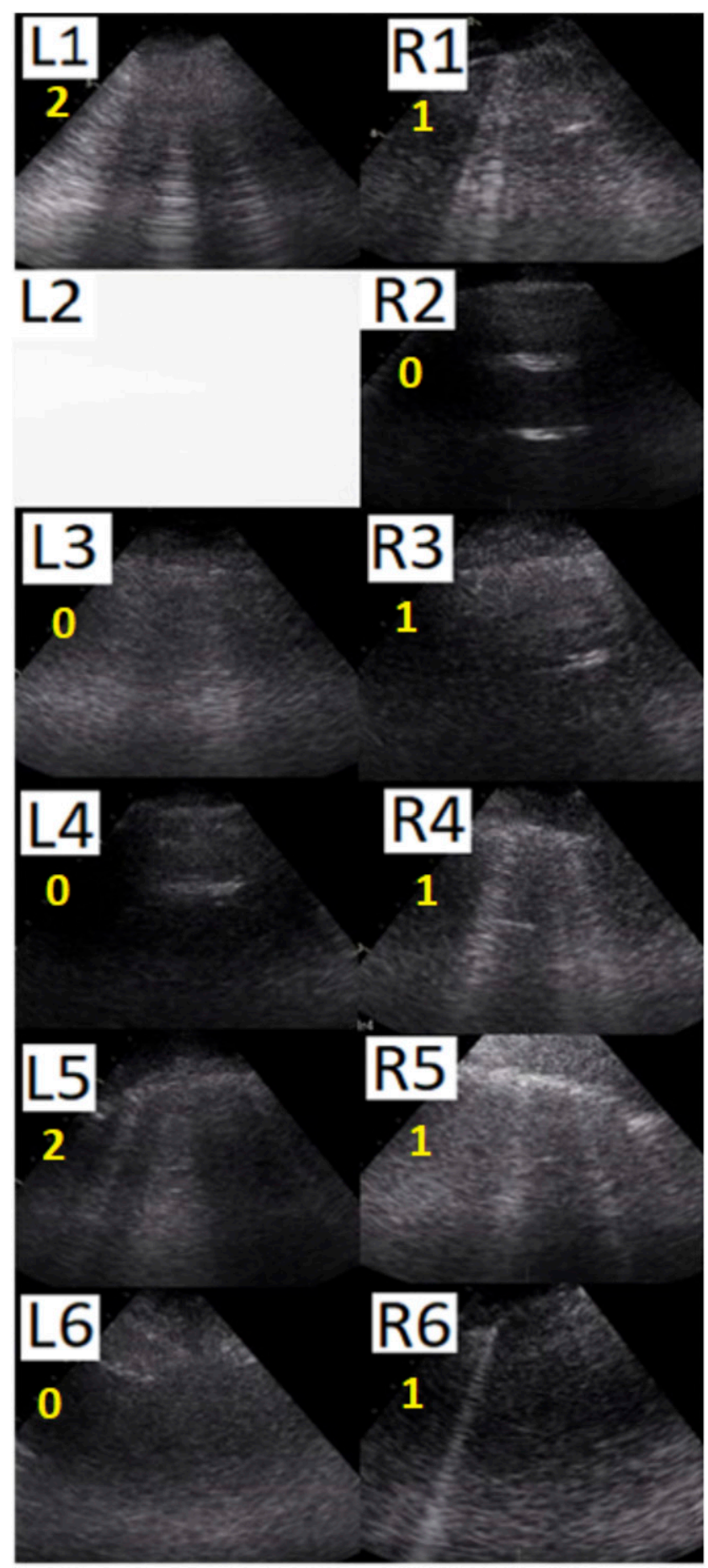

FIGURE 4. Lung ultrasound images for patient B. L= left hemithorax; $\mathrm{R}=$ right hemithorax; 1,2 = anterior thoracic areas; 3,4 = lateral thoracic areas; 5, $6=$ posterior thoracic areas.

permits the assessment of lung aeration immediately after any change in ventilator parameters. Although it would have been attractive to perform follow-up LUS in the prone position, it is impossible to score and, thus, compare the chest regions that are scored in the supine position.

It has been speculated that poor lung compliance in the context of COVID-19 could indicate a higher likelihood of extensive atelectasis and, possibly, positive response to recruitment maneuvers. ${ }^{17}$ Nevertheless, this hypothesis has not been confirmed by clinical data where it has been shown that lung compliance is unrelated to the extent of parenchymal involvement in patients with COVID-19. ${ }^{18}$ Both patients presented here had abnormal dynamic compliance of the respiratory system that did not improve to normal values despite clinical improvement. It is highly likely that our patient had decreased chest wall compliance because of severe obesity, which is a common comorbidity in severe COVID-19 pneumonia. This is further supportive of the simultaneous use of LUS and lung mechanics. A decrease in LUS aeration score without a concomitant improvement of compliance may imply overdistention of lung units, whereas slight changes in compliance without changes in the LUS score may imply positionrelated changes in chest wall compliance.

In conclusion, we report our experience with LUS in two COVID-19 patients with refractory hypoxia who required invasive ventilation and adjunctive therapies for refractory hypoxemia. The two presented cases suggest the need for a personalized approach in use of adjunctive therapies in patients with COVID-19. Lung ultrasound could be an attractive bedside tool to guide their use.

Received May 25, 2020. Accepted for publication September 2, 2020.

Published online September 9, 2020.

Acknowledgment: Publication charges for this article were waived due to the ongoing pandemic of COVID-19.

Authors' addresses: Charalampos Pierrakos, Department of Intensive Care, CHU Brugmann, Brussels, Belgium, and Department of Intensive Care, Amsterdam UMC, University of Amsterdam, Amsterdam, The Netherlands, E-mail: charalampos.pierrakos@chubrugmann.be. Rachid Attou, Patrick M. Honore, and David De Bels, Department of Intensive Care, CHU Brugmann, Brussels, Belgium, E-mails: rachid.attou@chu-brugmann.be, patrick.honore@ chu-brugmann.be, and david.debels@chu-brugmann.be. Enrica lesu and Hugues Baelongandi, Department of Anesthesiology, CHU Brugmann, Brussels, Belgium, E-mails: enrica.iesu@chu-brugmann.be and hugues.baelongandi@chu-brugmann.be. Lieuwe D. J. Bos, Department of Intensive Care, Amsterdam UMC, University of Amsterdam, Amsterdam, The Netherlands, Laboratory of Experimental Intensive Care and Anesthesiology (LEICA), Amsterdam UMC, University of Amsterdam, Amsterdam, The Netherlands, and Department of Respiratory Medicine, Amsterdam UMC, University of Amsterdam, Amsterdam, The Netherlands, E-mail: lieuwe.bos@gmail.com. Marcus J. Schultz, Department of Intensive Care, Amsterdam UMC, University of Amsterdam, Amsterdam, The Netherlands, Laboratory of Experimental Intensive Care and Anesthesiology (LEICA), Amsterdam UMC, University of Amsterdam, Amsterdam, The Netherlands, Mahidol-Oxford Tropical Medicine Research Unit (MORU), Mahidol University, Bangkok, Thailand, and Nuffield Department of Medicine, University of Oxford, Oxford, United Kingdom, E-mail: marcus.j.schultz@ gmail.com.

This is an open-access article distributed under the terms of the Creative Commons Attribution (CC-BY) License, which permits unrestricted use, distribution, and reproduction in any medium, provided the original author and source are credited.

\section{REFERENCES}

1. JHU, 2020. COVID-19 Dashboard by the Center for Systems Science and Engineering (CSSE) at Johns Hopkins University (JHU). Available at: https://gisanddata.maps.arcgis.com/apps/opsdashboard/index. html\#/bda7594740fd40299423467b48e9ecf6?utm_source=sn\& utm_medium=referral\&utm_content=null\&utm_campaign=BSLB 1_CA01_GL_BSLB_AWA_CA01_GL_LSGR_PubH_Coronovirus_ LandingPage. Accessed July 31, 2020. 
2. Guan W et al., 2020. Clinical characteristics of coronavirus disease 2019 in China. N Engl J Med 382: 1708-1720.

3. Schultz MJ, Sivakorn C, Dondorp AM, 2020. Challenges and opportunities for lung ultrasound in novel coronavirus disease (COVID-19). Am J Trop Med Hyg 102: 1162-1163.

4. Gattinoni L, Marini JJ, Quintel M, 2020. Recruiting the acutely injured lung: how and why? Am J Respir Crit Care Med 201: 130-132.

5. Chiumello D, Mongodi S, Algieri I, Vergani GL, Orlando A, Via G, Crimella F, Cressoni M, Mojoli F, 2018. Assessment of lung aeration and recruitment by CT scan and ultrasound in acute respiratory distress syndrome patients. Crit Care Med 46: 1761-1768.

6. Lomoro P, Verde F, Zerboni F, Simonetti I, Borghi C, Fachinetti C, Natalizi A, Martegani A, 2020. COVID-19 pneumonia manifestations at the admission on chest ultrasound, radiographs, and CT: single-center study and comprehensive radiologic literature review. Eur J Radiol Open 7: 100231.

7. Yasukawa K, Minami T, 2020. Point-of-care lung ultrasound findings in patients with COVID-19 pneumonia. Am J Trop Med Hyg 102: 1198-1202.

8. Sivakorn C, Luvira V, Muangnoicharoen S, Piroonamornpun $P$, Ouppapong T, Mungaomklang A, lamsirithaworn S, 2020. Case report: walking pneumonia in novel coronavirus disease (COVID19): mild symptoms with marked abnormalities on chest imaging. Am J Trop Med Hyg 102: 940-942.

9. Peng QY, Wang XT, Zhang LN, 2020. Findings of lung ultrasonography of novel corona virus pneumonia during the 2019-2020 epidemic. Intens Care Med 46: 849-850.

10. Constantin JM et al.; AZUREA Network, 2019. Personalised mechanical ventilation tailored to lung morphology versus low positive end-expiratory pressure for patients with acute respiratory distress syndrome in France (the LIVE study): a multicentre, single-blind, randomised controlled trial. Lancet Respir Med 7: 870-880.

11. Cavalcanti $A B$ et al., 2017. Effect of lung recruitment and titrated positive end-expiratory pressure (PEEP) vs low PEEP on mortality in patients with acute respiratory distress syndrome-a randomized clinical trial. JAMA 318: 1335-1345.

12. Acute Respiratory Distress Syndrome NetworkBrower RG, Matthay MA, Morris A, Schoenfeld D, Thompson BT, Wheeler $A, 2000$. Ventilation with lower tidal volumes as compared with traditional tidal volumes for acute lung injury and the acute respiratory distress syndrome. N Engl J Med 342: 1301-1308.

13. Bouhemad B, Brisson H, Le-Guen M, Arbelot C, Lu Q, Rouby JJ, 2011. Bedside ultrasound assessment of positive endexpiratory pressure-induced lung recruitment. $A m \mathrm{~J}$ Respir Crit Care Med 183: 341-347.

14. Rode $B$, Vučić $M$, Siranović $M$, Horvat $A$, Krolo $H$, Kelečić $M$, Gopčević A, 2012. Positive end-expiratory pressure lung recruitment: comparison between lower inflection point and ultrasound assessment. Wien Klin Wochenschr 124: 842-847.

15. House DR, Amatya Y, Nti B, Russell FM, 2020. Impact of bedside lung ultrasound on physician clinical decision-making in an emergency department in Nepal. Int J Emerg Med 13: 10-14.

16. Umuhire OF, Henry MB, Levine AC, Cattermole GN, Henwood P, 2019. Impact of ultrasound on management for dyspnea presentations in a Rwandan emergency department. Ultrasound J 11: 18.

17. Gattinoni L, Chiumello D, Caironi P, Busana M, Romitti F, Brazzi L, Camporota L, 2020. COVID-19 pneumonia: different respiratory treatment for different phenotypes? Intens Care Med 46: 1099-1102.

18. Bos LD, Paulus F, Vlaar APJ, Beenen LFM, Schultz MJ, 2020. Subphenotyping ARDS in COVID-19 patients: consequences for ventilator management. Ann Am Thorac Soc 17:1161-1163. 